\title{
Estilos de afrontamiento y nivel de estrés de madres adolescentes con hijos hospitalizados en la unidad de cuidados intensivos neonatales, Chiapas, México
}

\section{Beatriz E. López-Mayorga1 , Beatriz Carmona-Mejía1 , Gandhy Ponce-Gómez³}

\begin{abstract}
Introduction: adolescent pregnancy is considered a public health problem due to the adverse effects that the mother and the child can have during pregnancy, childbirth and puerperium; complications that are attributed to the adolescent's age, psychological implications and biological factors. Among the main problems for the child are prematurity, delayed uterine growth and respiratory distress syndrome. The teen has to take on the new rol, and practice coping (COPING); mothers simultaneously experience stress.

Objective: discuss the coping styles and stress level of adolescent mothers with children hospitalized in the NICU neonatal intensive care unit.

Material and methods: quantitative, cross-sectional, observational, prolect study. Sampling at convenience with 80 selected teens in a second-level hospital; a Likert-type self-report questionnaire was implemented consisting of sociodemographic data, adolescent coping scale and parental stress scale, both instruments adapted by López, Carmona and Ponce in 2016. Type of analysis: SPSS 23, and descriptive statistics, inferential statistical.

Results: regarding coping styles, teenage girls were found to be targetins the unproductive style, the self-incriminate strategy. In relation to the analysis of perceived stress, it was found at high levels, the subscale corresponding to the relationship with the child and the maternal role, shows the average stress, in the subdimension are the aspects and sounds of the unit and appearence and behavior of the child.

Conclusion: adolescent mothers are in the style of unproductive coping often distrust their abilities, causing feelings of insecurity, thus hospitalizing the newborn in the NICU and the relationship with the child and the maternal role for them is extremely stressful . Therefore, educational intervention and training are requiered to direct your behaviors to a positive approach and thus improve the performance regarding the care of your child in neonatal intensive therapy.
\end{abstract}

Key words: coping, parental stress, adolescent mothers.

Citación: López-Mayorga B., Carmona-Mejía B., Ponce-Gómez G. "Estilos de afrontamiento y nivel de estrés de madres adolescentes con hijos hospitalizados en la unidad de cuidados intensivos neonatales, Chiapas, México". Rev Enferm Neurol. 2019;18(1): pp. 41-51.

Correspondencia: Gandhy Ponce-Gómez

Email: gandhy_ponce@yahoo.com.mx

${ }^{1}$ Hospital Regional Dr. Rafael Pascacio Gamboa, ISSSTE. ${ }^{2}$ Prof. de Carrera Asoc. C. Tiempo Completo Carrera de Enfermería. Facultad de Estudios Profesionales Zaragoza-UNAM.
${ }^{3}$ Prof. de Carrera Titular A. Tiempo Completo. Escuela Nacional de Enfermería y Obstetricia-UNAM.

Recibido: 21 de febrero 2018.

Aceptado: 20 de agosto 2018.

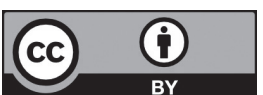




\section{Resumen}

Introdución: el embarazo en adolescentes se considera un problema de salud pública por los efectos adversos que pueden tener la madre y el niño durante todo el embarazo. Entre los principales problemas para el niño se destaca prematurez, retraso en el crecimiento y síndrome de dificultad respiratoria. La adolescente tiene que asumir el nuevo rol, y practicar el afrontamiento (COPING); simultáneamente las madres experimentan estrés.

Objetivo: analizar los estilos de afrontamiento y nivel de estrés de madres adolescentes con hijos hospitalizados en la unidad de cuidados intensivos neonatales UCIN.

Material y métodos: estudio cuantitativo, transversal, observacional, prolectivo. Muestreo a conveniencia con 80 adolescentes seleccionadas en un hospital de segundo nivel; se aplicó un cuestionario de autoinforme en escala tipo Likert integrado por datos sociodemográficos, escala de afrontamiento en adolescentes y escala de estrés parental ambos instrumentos adaptados por López, Carmona y Ponce en 2016. Tipo de análisis: SPSS 23, y estadística descriptiva, estadística inferencial.

Resultados: respecto a los estilos de afrontamiento se encontró que las adolescentes se dirigen al estilo improductivo, la estrategia autoinculparse. En relación al análisis del estrés percibido, se encontró en niveles altos, la subescala correspondiente a la relación con el niño y el rol materno, muestra el estrés medio, en la subdimensión se tienen los aspectos y sonidos de la unidad y la apariencia y conducta del niño.

Conclusiones: las madres adolescentes se dirigen al estilo de afrontamiento improductivo suelen desconfiar de sus capacidades, provocando sentimientos de inseguridad, de esta manera la hospitalización del recién nacido en la UCIN y la relación con el niño y el rol materno para ellas es en extremo estresante. Por lo tanto, se requieren de una intervención educativa y de capacitación para direccionar sus conductas hacía un afrontamiento positivo y de esta manera mejorar la actuación respecto al cuidado de su hijo en una terapia intensiva neonatal.

Palabras claves: afrontamiento, estrés parental, madres adolescentes.

\section{Introducción}

México es el país de la Organización para la Cooperación y el Desarrollo Económicos (OCDE) con la tasa más alta de madres adolescentes, entre 15 y 19 años de edad. ${ }^{1}$ El embarazo en adolescentes ocurre dentro los dos primeros años de edad ginecológica entendiéndose por tal, el tiempo transcurrido desde la menarquía o cuando ellas tienen la total dependencia social y económica de la familia parental, se trata de mujeres que por su configuración anatómica y psicológica aún no han alcanzado la madurez necesaria para cumplir el rol de madres.

Este hecho ha sido descrito como un problema de salud pública en Latinoamérica dada su magnitud y las repercusiones biológicas, psicológicas y sociales que conlleva. A nivel biológico, el riesgo de morbimortalidad 
es elevado cuanto más joven sea la madre más complicaciones durante el embarazo, aunado a la pobreza de su país de residencia; el parto y el posparto. A nivel psicológico las jóvenes no se encuentran preparadas para asumir la responsabilidad de este nuevo rol. A nivel social, la joven verá limitadas las posibilidades para el futuro por la posible interrupción de su educación formal.

De acuerdo con la información proporcionada por la Encuesta Nacional de la Dinámica Demográfica (ENADID) del 2014 México, reportó por cada 1000 mujeres adolescentes 77 nacimientos, ${ }^{2}$ en este contexto el Estado de Chiapas registro 93 nacimientos ocupando uno de los primeros cinco lugares a nivel nacional. ${ }^{3}$

A su vez, el Hospital de la Mujer "Dr. Rafael Pascasio Gamboa" durante el 2015 registró 11,859 partos, de los cuales 2,865 corresponden a mujeres menores de 19 años, e ingresaron a la Unidad de Cuidados Intensivos Neonatales (UCIN) 930 recién nacidos producto de adolescentes.

Por otro lado, la mayoría de las niñas que se embarazan viven en zonas rurales, las tasas de natalidad son más altas donde prevalece el matrimonio infantil, y estos suelen ser más frecuentes donde hay pobreza extrema, las adolescentes que pertenecen a una etnia o a un grupo marginado son más vulnerables a quedar embarazadas, no tienen opción y tampoco oportunidades en la vida, o tienen un acceso limitado o nulo a la salud sexual y reproductiva..$^{2-5}$

En la actualidad, existen pocos estudios relacionados con el estilo de afrontamiento y la relación con el estrés en madres adolescentes con hijos hospitalizados en la UCIN.

La hospitalización de un bebé en la UCIN constituye un suceso estresante, algunas investigaciones lo han caracterizado como un hecho traumático para los padres debido a que el pronóstico de salud del recién nacido puede ser incierto; se acrecientan los miedos, culpas, desilusión, enojo, sufrimiento, entre otros sentimientos; al mismo tiempo, que la adolescente se encuentra inmersa ante esta situación necesitará asumir responsabilidades en momentos inadecuados para hacer frente a la maternidad, siendo este un proceso difícil dónde para confrontar el problema deberá hacer uso de sus recursos llamados estilos de afrontamiento que le permitirán dar respuesta a las situaciones que le generen estrés. ${ }^{4,6-8}$

El objetivo del estudio fue identificar los estilos de afrontamiento que utiliza la madre adolescente para enfrentar la crisis, así como determinar las fuentes de estrés identificadas por la madre adolescente ante la hospitalización de su hijo en la UCIN.

\section{Afrontamiento y estrés ante la hospitali- zación}

El afrontamiento desempeña un papel de importante mediador entre las experiencias estresantes a las que están sometidas las personas, los recursos personales y sociales con los que cuentan para hacerles frente y las consecuencias que se derivan para la salud física y psicológica. El afrontamiento se deriva de las interacciones de las personas con múltiples circunstancias de su vida en los contextos socioculturales, lo cual deja ver la multicausalidad del fenómeno.

Frydenberg y Lewis creadores de la escala de afrontamiento para adolescentes (ACS) usada en este estudio, definen el afrontamiento como: "un grupo de acciones, cogniciones y respuestas afectivas que surgen como reacción a un nuevo evento particular. Estas respuestas representan un intento por restaurar el equilibrio o resolver 
una perturbación del individuo. Esto puede lograrse a través de la solución del problema (esto es, modificar el estímulo) o acomodarse a las circunstancias sin tratar de lograr una solución".

La clasificación del afrontamiento en adolescentes propuesta por estos autores consiste en 18 estrategias obtenidas empíricamente y que se agrupan en tres estilos básicos: dirigido a la resolución del problema (dirigido al problema, trabajar para resolver el problema), afrontamiento emocional en relación con los demás (en referencia a los otros, por ejemplo, buscar apoyo social) y afrontamiento improductivo (culpa y preocupación).

El afrontamiento es una forma de manejar situaciones que puedan generar estrés en el individuo, con las que busca tolerar, minimizar, aceptar o ignorar aquello que sobrepasa sus capacidades, ya sea resignificando el estímulo, a través de las acciones, pensamientos, afectos y emociones que el individuo utiliza a la hora de abordar la situación desbordante. Por tal motivo, forma parte de los recursos psicológicos o psicosociales que el individuo utiliza para hacer frente a situaciones estresantes, ejerce un efecto de mediación en los rasgos de personalidad. ${ }^{10}$

De la misma manera, Lazarus y Folkman señalan que "el estrés psicológico es el resultado de una relación entre el sujeto y el entorno, que es evaluado por éste como amenazante o desbordante de sus recursos y que pone en peligro su bienestar". Identificaron los sucesos de vida en la adolescencia en términos de eventos mayores, desencadenantes de estrés, asociados con la presencia de problemas emocionales y de salud (como la muerte de un familiar, embarazarse o casarse) y en términos de eventos cotidianos (como discusiones frecuentes entre los padres y hermanos) que pueden convertirse en mayores o críticos, y en este sentido, ser estresores crónicos dada su frecuencia, intensidad o duración, generando ansiedad, e incrementando sus niveles al grado de provocar problemas en las salud integral del adolescente. ${ }^{11}$

De acuerdo con Seiffge-Krenke, los individuos se vuelven más vulnerables y propensos al estrés durante periodos de transición biológica, social y psicológica. Es así como en la adolescencia, etapa del desarrollo en la cual se deben confrontar un gran número de cambios, los jóvenes pueden encontrarse bajo circunstancias personales, sociales, políticas y económicas que les generen situaciones estresantes. ${ }^{12}$

En la revisión de la literatura se detectó que en las adolescentes embarazadas y no embarazadas, se reporta que las diferencias significativas entre las estrategias utilizadas entre un grupo y otro son: buscar apoyo social, invertir en amigos íntimos y fijarse en lo positivo a favor del grupo de adolescentes no embarazadas. ${ }^{13}$

Mientras que en las subescalas a favor del grupo de embarazadas son: hacerse ilusiones, falta de afrontamiento, auto inculparse y buscar apoyo profesional, lo que significa que el estilo de afrontamiento en este grupo es centrado en la emoción, por lo que se podría suponer que continuará con la misma forma cuando el bebé haya nacido. ${ }^{14,15}$

En este sentido, las madres con hijos hospitalizados en la UCIN presentan estrés derivado de la imposibilidad de ejercer su rol de padres, ya que la hospitalización forzosa de un niño o niña y los horarios restringidos, son una situación de crisis que provoca reacciones emocionales como: desilusión, tristeza, depresión, hostilidad, enojo, miedo, ansiedad, sentimiento de fracaso y pérdida de autoestima. ${ }^{16,17}$

Miles, et al., explican que muchos aspectos de la UCIN son estresantes para 
los padres, incluyendo las hospitalizaciones prolongadas, exposición a un ambiente implementado con máquinas, y la apariencia de su pequeño y frágil recién nacido; concluyen que la alteración en el rol parental causada por la enfermedad del RN, es la situación que genera más estrés. La segunda área más importante que lo genera está relacionada con la apariencia y comportamiento del RN. ${ }^{18}$

Algunos estudios reportaron como poco estresantes los aspectos y sonidos de la unidad y apariencia y conducta del niño. ${ }^{19,20}$

Una investigación realizada en padres con niños operados de cardiopatías congénitas, evidenció que los factores de estrés parental se agruparon en factores derivados del paciente, de los padres, del ambiente y del equipo clínico. La pérdida del rol parental, la apariencia física y el comportamiento del hijo fueron identificados como los más estresantes. ${ }^{21}$

\section{Material y métodos}

Se trata de un estudio Cuantitativo, de tipo transversal, observacional prolectivo y comparativo en una muestra de 63 sujetos calculada con la fórmula para poblaciones cuantitativas finitas y constituida por mujeres adolescentes (rangos de edad 12 a 19 años) con hijos hospitalizados en la unidad de cuidados intensivos neonatales de un hospital de tercer nivel de atención en Tuxtla Gutiérrez, Chiapas.

\section{Las variables de estudio fueron:}

a.Afrontamiento: definida como forma o modo con el que la persona encara cualquier situación (general) y afronta una problemática particular. ${ }^{9}$ Cuyas dimensiones implican la resolución del problema; b. relación con los demás, y c. el afrontamiento improductivo.

Estrés: experiencia que ha causado un sentimiento de ansiedad, molestia o tensión. ${ }^{18}$

Previa autorización por el Comité de Enseñanza e Investigación de la institución seleccionada para el estudio y en coordinación con el departamento de trabajo social se abordaron a las madres adolescentes durante los horarios de pase de lista, con el fin de lograr un ambiente de privacidad y comodidad se aplicaron los instrumentos durante sábado y domingo en las oficinas de trabajo social, se les explicó el objetivo de la investigación. De igual manera, les fue solicitada la participación voluntaria, después, se procedió a firmar el consentimiento informado para formalizar su participación

\section{Instrumento de medición}

El cuestionario estuvo integrado por tres apartados; el primero: incluye las variables sociodemográficas de la madre y neonato; el segundo: por el instrumento para evaluar el afrontamiento (ACS, Adolescent Coping Scale) y el tercer apartado la escala de estrés parental (PSS: NICU Neonatal Intensive Care Unit) ${ }^{22}$ adaptados por López, Carmona y Ponce 2016.

Las variables sociodemográficas de las madres recopilan antecedentes sociodemográficos en el caso de la madre: edad, estado civil, con quién vive, escolaridad, ocupación, religión, procedencia, ingreso económico y número de hijos; con respecto al neonato: días de hospitalización, diagnóstico médico, edad gestacional, apoyo ventilatorio.

El Adolescent Coping Scale (ACS-2) de Frydenberg y Lewis, ${ }^{23}$ está integrado por 18 estrategias que corresponden a tres estilos (afrontamiento dirigido a la resolución del problema, en relación con los demás y el improductivo), 
compuesto por 80 elementos; que se puntúan mediante una escala de tipo Likert de 5 puntos que abarca desde "no se me ocurre nunca o no lo hago" (puntuación 1), hasta "me ocurre o lo hago con mucha frecuencia" (puntuación 5). Reportando un alfa global de Cronbach para la población mexicana entre .85 y .95 . Se sometió a traducción, adaptación cultural y validación de contenido, constituido por 17 estrategias con un total de 51 ítems alcanzando un alpha de .803 a la aplicación del instrumento.

La escala de estrés parental (EEP) adaptado del Neonatal Intensive Care Unit (PSS: NICU) para evaluar el estrés de los padres relacionado con la hospitalización de un hijo, está vez, neonatal. Se sometió a traducción, adaptación cultural y validación de contenido por jueceo de expertos, constituido de 26 ítems en tres subescalas medido con una escala de medición tipo Likert del 1 al 5, donde 1 significa cuando lo descrito en el ítem no es tan estresante y 5 es extremadamente estresante. El alpha de Cronbach alcanzó un nivel de .944 en la aplicación final

Aspectos y sonido de la unidad: analiza el nivel de estrés percibido por los padres en relación al ambiente de la UCIN tales como: la presencia de monitores y equipo, los ruidos constantes de los monitores y equipo, los sonidos repentinos de los monitores, los otros bebés enfermos en la unidad, la cantidad de gente trabajando en la unidad.

Apariencia y comportamiento del bebé: se refiere al aspecto en la que se encuentra el niño físicamente y a los cuidados que el mismo requiere: color y tamaño del bebé, conexiones y aparatos externos, gesticulaciones, la presencia o no de llanto y movimientos repentinos, entre otros.
Alteración del rol parental: abarca diversos aspectos referidos a la alteración de los vínculos entre madre/padre y su bebé, debido a su condición de internación (aislamiento, no poder procurarle cuidados, contacto estrecho, etcétera).

Respecto a las consideraciones éticas practicadas, se tuvo apego a los principios de justicia, beneficencia y no maleficencia respetando la confidencialidad de los datos de los participantes en este caso exclusivamente para fines de esta investigación, en apego de la Ley General de Salud para la Materia de Investigación. Se firmó el consentimiento informado después de una explicación clara y completa de participación y uso de los datos con fines de investigación.

\section{Resultados}

La población de estudio fue de 63 madres adolescentes con hijos hospitalizados en la UCIN, en cuanto a edad predominó la adolescencia tardía con el $92 \%$, siendo la edad media de los sujetos estudiados de $17.5 \pm 1.6$ años; respecto al lugar de procedencia se encontró que el 50.8\% radica en la zona urbana y el resto en la zona rural, en cuanto al tipo de embarazo predominó el planeado con el $49.2 \%$.

El 84\% de las participantes están casadas, el resto son madres solteras, de ellas el $46 \%$ viven en un espacio independiente a sus familiares; el grado de estudios predominante fue nivel básico con el 62\%; de igual forma, se observó que el $79 \%$ se dedican a ser amas de casa y 4 de cada 10 adolescentes tienen un ingreso mensual familiar entre 2,125.00 y 3,249.00 pesos (esto muestra que las adolescentes de esta investigación se encuentran en la clasificación de pobreza extrema). 
Las siguientes estrategias son las más representativas del grupo (se citan en orden decreciente de acuerdo a la frecuencia de uso): 1. fijarse en lo positivo, 2. buscar apoyo espiritual, 3. auto inculparse, 4. ignorar el problema y reservarlo para sí, y 5. falta de afrontamiento y reducción de la tensión. Por otro lado, el estilo de afrontamiento que distinguen a las adolescentes para enfrentar la hospitalización de su recién nacido es el improductivo, como puede observarse 5 de 7 estrategias correspondiente a este estilo son utilizadas, le sigue el dirigido a la relación con los demás, y el menos utilizado, la resolución del problema (tabla1).

Tabla 1. Estilos y estrategias de afrontamiento de madres adolescentes.

\begin{tabular}{|c|c|c|}
\hline & $M$ & DE \\
\hline Estilo dirigido a la resolución del problema & 2.53 & .51 \\
\hline Buscar diversiones relajantes & 2.0 & .62 \\
\hline Distracción física & 2.1 & .79 \\
\hline Fijarse en lo positivo & 3.9 & .91 \\
\hline Concentrarme en resolver el problema & 3.1 & .81 \\
\hline Esforzarse en tener éxito & 2.2 & .47 \\
\hline Estilo dirigido a la relación con los demás & 3.10 & .73 \\
\hline Buscar apoyo espiritual & 3.7 & .99 \\
\hline Buscar ayuda profesional & 2.9 & .59 \\
\hline Buscar pertenencia & 3.2 & .95 \\
\hline Buscar apoyo social & 2.9 & .82 \\
\hline Acción social & 2.7 & .57 \\
\hline Estilo improductivo & 3.16 & .95 \\
\hline Hacerse ilusiones & 2.5 & .90 \\
\hline Falta de afrontamiento & 3.3 & .90 \\
\hline Reducción de la tensión & 3.3 & 1.37 \\
\hline Ignorar el problema & 3.4 & 1.05 \\
\hline Autoinculparse & 3.6 & 1.06 \\
\hline Reservarlo para si & 3.4 & .87 \\
\hline Preocuparse & 2.7 & .7 \\
\hline
\end{tabular}

n=63 Puntaje mínimo 1, máximo 4; puntaje mayor de 4 indica estilo con mayor frecuencia utilizada. 
En relación al estrés percibido se observó en primer término, que las madres adolescentes perciben la internación de sus hijos en la UCIN como un suceso moderadamente estresante $(\mathrm{M}=3.19$, $\mathrm{DE}=.899)$.

Destacaron los sucesos cuando parece que mi bebé tiene dolor, ver agujas y tubos puestos en mi bebé y cuando mi bebe se ve triste; de la dimensión aspectos y sonidos de la UCIN ( $\mathrm{M}=2.54, \mathrm{DE}=1.03)$ destacaron los ruidos constantes de aparatos y equipo y los sonidos repentinos de los aparatos (tabla 2).

Tabla 2. Promedio de estrés de madres adolescentes con hijos hospitalizados en la UCIN

\begin{tabular}{|c|c|c|}
\hline & M & $D E$ \\
\hline Aspectos y sonidos de la unidad & 2.54 & 1.00 \\
\hline La presencia de aparatos y equipos & 2.5 & 1.29 \\
\hline Los ruidos constantes de aparatos y equipos & 2.8 & 1.32 \\
\hline Los sonidos repentinos de los aparatos & 2.6 & 1.28 \\
\hline Los otros bebés enfermos en el lugar & 2.5 & 1.35 \\
\hline La cantidad de gente trabajando en el lugar & 2.2 & 1.77 \\
\hline Apariencia y comportamiento del bebé & 3.1 & 1.03 \\
\hline Tubos y equipos cerca de mi bebé & 3.2 & 1.51 \\
\hline Heridas, cortes o incisiones en mi bebé & 3.2 & 1.58 \\
\hline El color extraño de mi bebé & 2.8 & 1.51 \\
\hline Respiración extraña o anormal en mi bebé & 3.3 & 1.47 \\
\hline El tamaño pequeño de mi bebé & 2.7 & 1.38 \\
\hline La apariencia arrugada de mi bebé & 2.0 & 1.18 \\
\hline Mi bebe conectado en una máquina & 3.2 & 1.62 \\
\hline Ver agujas y tubos puestos en mi bebé & 3.6 & 1.37 \\
\hline Cuando parece que mi bebé tiene dolor & 3.7 & 1.31 \\
\hline Cuando mi bebé se ve triste & 3.6 & 1.34 \\
\hline La apariencia débil de mi bebé & 3.4 & 1.67 \\
\hline Movimientos repentinos y sin descanso de mi bebé & 2.8 & 1.57 \\
\hline Mi bebé no llora como los otros bebés & 2.8 & 1.54 \\
\hline Alteración del rol materno & 3.7 & 1.09 \\
\hline Estar separada de mi bebé & 3.8 & 1.48 \\
\hline No alimentar a mi bebé personalmente & 3.8 & 1.34 \\
\hline No cuidar a mi bebé personalmente & 3.4 & 1.48 \\
\hline No poder abrazar a mi bebé cuando yo quiera & 3.8 & 1.39 \\
\hline Sentirme incapaz de proteger a mi bebé del dolor & 3.9 & 1.42 \\
\hline Sentirme incapaz de proteger a mi bebé durante este tiempo & 3.9 & 1.33 \\
\hline No tener tiempo a solas con mi bebé & 3.7 & 1.44 \\
\hline
\end{tabular}

n=63 Puntaje mínimo de 1, máximo 5 "mayor puntuación indica las fuentes de estrés identificadas por las madres adolescentes 
Respecto al afrontamiento se encontró que existe una asociación lineal baja directamente proporcional y en estadística significativa entre el afrontamiento y los días de hospitalización de niños de madres adolescentes con hijos hospitalizados en la UCIN se corrió la prueba de asociación de Pearson. Reveló una relación débil entre más días de hospitalización mayor afrontamiento; esto explica al menos el $30 \%$ de los casos en que existe mayor afrontamiento en madres con hijos con mayor número de días de hospitalización $(\mathrm{rp}=.362 \mathrm{p}=.004)$. De igual forma, al realizar la prueba entre el nivel de estrés y días de hospitalización no se encontró asociación y diferencias en estadística significativas entre ambas variables $(\mathrm{rp}=.022 \mathrm{p}>0.05)$, esto denota que el nivel de estrés de las adolescentes no se relaciona con los días de hospitalización de su recién nacido

\section{Discusión.}

Las estrategias utilizadas con mayor frecuencia por las adolescentes, son: (se mencionan de mayor a menor) autoinculparse, ignorar el problema, reservarlo para sí, reducción de la tensión y falta de afrontamiento (estilo improductivo), siendo este resultado similar con otros estudios; donde el objetivo fue estudiar la relación entre estilos y estrategias de afrontamiento en adolescentes embarazadas, describen las estrategias utilizadas por las mujeres adolescentes ante estresores: hacerse ilusiones, culparse a sí misma, reducción de la tensión y falta de afrontamiento (estilo improductivo). ${ }^{15}$

Además, la percepción del estrés en este estudio ha demostrado que la internación en la UCIN resulta ser un hecho moderadamente estresante para las madres, siendo la alteración del rol materno la dimensión ubicada en un nivel extremo para las participantes. Estos resultados coinciden con otros estudios, la separación con el bebé y la imposibilidad de llevar adelante los cuidados parentales esperados, se constituyen en la situación más estresante para los mismos. El ambiente de la UCIN preocupa algo más que el comportamiento y apariencia del bebé, y este último preocupa más a las madres que a los padres. $^{24,25}$

Por último, el internamiento de un hijo en la UCIN es reconocida como una crisis vital y estresante para la madre, de tal manera que, cuando esto sucede ellas pueden sentirse incapaces de abordar circunstancias nuevas, en este sentido las adolescentes con hijos hospitalizados deben realizar un gran esfuerzo para poder afrontar de forma positiva la situación, tomar en cuenta las condiciones propias de la etapa como inestabilidad emocional, a su vez la maternidad genera una serie de privaciones que refuerzan la situación de pobreza y restringen el proyecto de vida de este grupo vulnerable.

Las fuentes extremadamente estresantes en la unidad de cuidados intensivos neonatales identificadas por las adolescentes correspondieron a la apariencia y comportamiento del bebé y el no poder cumplir con el rol de madre por el aislamiento al que se somete al niño; de la misma forma, se visualizaron como factores influyentes: el nivel educativo básico, no profesar ninguna religión, ser madre soltera y tener un embarazo forzado (ANOVA $\mathrm{p}<.005$ ).

\section{Conclusion}

Esta investigación, aporta evidencias trascendentes sobre los estilos de afrontamiento como un recurso para hacer frente a situaciones estresantes como resultado de los estímulos del entorno, así como la capacidad que tiene el ser humano 
para confrontarlas, en este sentido podemos conocer las necesidades de las adolescentes cuando enfrentan una situación de crisis, tal es el caso del internamiento de su hijo. Por este motivo, sobresale la necesidad de realizar desde la profesión de enfermería un trabajo de soporte que permita conocer los estilos y estrategias de afrontamiento para las madres adolescentes, en consecuencia, por medio del conocimiento en el tema, se direccione la atención a este grupo vulnerable, y ellas tengan herramientas de afrontamiento positivas a través de las cuales puedan disponer de respuestas ajustadas a las situaciones de estrés ante la hospitalización de su recién nacido.

\section{Referencias}

1. OCDE. Panorama de la educación: Indicadores de la OCDE. [Sitio en Internet] [Consultado: 13 septiembre 2018] Disponible en: http:// www.oecd.org/education/Mexico-EAG2014Country-Note-spanish.pdf

2. Fondo de Naciones Unidas para la Infancia (UNICEF) [Internet]. Hechos sobre adolescencia y jóvenes en América Latina y el caribe. [Acceso: 30 mayo 2017] Disponible en: http://www.unicef.org/lac/Fast_facts_SP(1).pdf

3. UNICEF. Vivencias y relatos sobre el embarazo en adolescentes: una aproximación a los factores culturales, sociales y emocionales a partir de un estudio de seis países de la región. Informe de un grupo de investigadores de la UNICEF. Panamá: Plan y UNICEF;2014. Informe final. ) [Internet]. [Acceso: 30 septiembre 2018]. Disponible en: https://www.unicef.org/ecuador/embarazo_ adolescente_5_0_(2).pdf

4. United Nations Population Found (UNFPA) [nternet]. Maternidad en la niñez: enfrentar el reto del embarazo en adolescentes. Estado de la población mundial 2013. [Acceso: 3 junio 2016] disponible en: http://www.unfpa.org.mx/ publicaciones/SP-SWOP2013.pdf
5. UNFPA México [Internet]. Salud sexual y reproductiva en adolescentes y jóvenes 2016. [Acceso: 25 noviembre 2017]. Disponible en: http://www.unfpa.org.mx/ssr_adolescentes.php

6. UNICEF [Internet]. La adolescencia una época de oportunidades. Estado mundial de la infancia 2011. [Acceso: 25 noviembre 2016].

Disponible en: https://www.unicef.org/ spanish/sowc2011/pdfs/SOWC-2011-MainReport_SP_02092011.pdf

7. Lazarus, $\mathbf{R}$. The person-environment relationship, motivation, and coping. En: $\mathrm{R}$ Lazarus. Emotion and adaptation. $1^{\mathrm{a}}$ ed. New York: Oxford University Press.1991. p. 112-26

8. Seiffge-KI, Aunola K, Nurmi, J. Changes in stress perception and coping during adolescence: the role of situational and personal factors. 2009;80(1):259-79.

9. Frydenberg E, Lewis R. Things don't better just because you're older: a case for facilitating reflection. Brit J Educ Psychol 1999;69:81-94.

10. Burgos, Carretero, Elkington, PascualMarssetin \& Lobaccaro 2000; The role of personality, coping style and social support in health-related quality of life in HIV Infection. Quality of Life Research, 2000;9(4):423-37.

11. Seiffge-Krenke, I. Adolescents health. A developmental perspective. EUA. Lawrence Erlbaum. 1998.

12. Rubiños MA. Estrés, ansiedad y estilos de afrontamiento de padres con hijos internos en unidad de cuidados intensivos. Rev Cien Soc Humanidades Tecnol 2012;1(1):15-33.

13. Della MM. Estrategias de afrontamiento (coping) en adolescentes embarazadas escolarizadas. Rev Iber Educ disponible en: http:/ / rieoei.org/index. php

14. Zambrano PG, Vera LS, Flores OL. Relación entre funcionalidad familiar y las estrategias de afrontamiento utilizadas por adolescentes embarazada. Cien Cuida 2012;9(2):9-16.

15. Caruso A, Mikulic Isabel M. El estrés en padres de bebés prematuros internados en la unidad de cuidados intensivos neonatales: traducción y adaptación de la escala parental stressor 
scale: neonatal intensive care unit. An Investig 2012;19(2).

16. González ED, Ballesteros CN, Serrano RM. Determinantes estresores presentes en las madres de neonatos pretérmino hospitalizados en las unidades de cuidados intensivos. Cien Cuida 2012;9(1):43-53.

17. Miles MS, Mathes M. Preparation of parents for the ICU experience: what are we missing? Child Health Care 1991;20(3):132-7. DOI: http:/ / dx.doi.org/10.1207/s15326888chc2003_1

18. Lia RA, Ceriani CJ, Cravedi V, Rodriguez D. Estrés y depresión en madres de prematuros: un programa de intervención. Arch Arg Pediatr 2012;103(1):36-45.

19. Leonetti CL, Martins LM. Ansiedad materna en el periodo prenatal y posnatal: revisión de literatura. Rev Latino-am Enfermagem 2007;15(4). disponible en: http://www.scielo.br/ pdf/rlae/v15n4/es_v15n4a24.pdf
20. Ramirez LM, Pino AP, Springmuller PD, Claveria RC. Estrés en padres de niños operados de cardiopatías congénitas. Arch Argent Pediatr 2014;112(3):263-7.

21. Miles MS, Funk Sg, Carlson J. Parental stressor scale: neonatal intensive care unit. Nat Cent Biotechnol Inform 1993;42(3):148-52.

22. Frydenberg E, Lewis R. Adolescent coping scale second edition (ACS-2) [on line] [Acceso: 25 noviembre 2017]. Disponible en: https://www. acer.org/files / adolescent-coping-scale-2-longform-kylie-sample.pdf

23. Shaw RJ, Bernard RS, Storfer-Isser A, Rhine W, Horwitz SM. Parental coping in the neoantal intensive care unit. J Clin Psychol Med Sett 2013; 20(2):135-42.

24. Trombini SK, Helena SA, Veronez M, Harumi HL, Silva MS. A primeira visita ao fiho internado na unidade de terapia intensiva neoantal: percepcao dos países. Esc Anna Nery 2012;16(1):73-8. 